\title{
Design of Environmental Sensor Networks using Evolutionary Algorithms
}

\author{
Ferry Susanto $^{1,2}$, Setia Budi ${ }^{2,3}$, Paulo de Souza Jr. ${ }^{2}$, Ulrich Engelke ${ }^{2}$, and Jing $\mathrm{He}^{1}$ \\ ${ }^{1}$ College of Engineering and Science, Victoria University, Footscray VIC 3011, Australia \\ ${ }^{2}$ Data61, CSIRO, College Road, Sandy Bay TAS 7005, Australia \\ ${ }^{3}$ School of Engineering and Information \& Communication Technology, University of Tasmania, Sandy Bay TAS
}

7005, Australia

Email: \{ferry.susanto,jing.he\}@vu.edu.au, setia.budi@utas.edu.au,

\{ferry.susanto, budi.budi, paulo.desouza, ulrich.engelke\}@csiro.au

\begin{abstract}
An evolutionary algorithm (EA) assisted spatial sampling methodology is proposed to assist decision makers in Sensor Network (SN) deployments. We incorporated an interpolation technique with leave-one-out cross-validation (LOOCV) to assess the representativeness of a particular SN design. For validation of our method, we utilised the Tasmania's South Esk Hydrological Model developed by the CSIRO, which includes a range of environmental variables describing the landscape. We demonstrated that our proposed methodology is capable of assisting in initial design of SN deployment. Ordinary Kriging is shown to be the best suited spatial interpolation algorithm for the EA's LOOCV under the current empirical study.
\end{abstract}

Keywords - sensor network deployment, spatial sampling, optimisation, multi-objective, evolutionary algorithm, leaveone-out cross-validation, spatial data interpolation, ordinary kriging, inverse distance weighting, thin plate spline.

\section{INTRODUCTION}

Deploying Sensor Networks (SN) from ground-up has never been a simple task without knowledge of historical environmental information within the landscape. Random distribution of nodes does not necessarily establish a fit-to-purpose network. Whilst adding more sensor nodes within the Regionof-Interest (ROI) is likely to enhance the data usage and robustness of the $\mathrm{SN}$, it would also introduce undesirable increase in both deployment and maintenance costs. Careful design is therefore a critical process prior to the deployment of SN. It is one of the most significant factors in ensuring that the network delivers fit-for-purpose data in a cost-effective way. Two fundamental questions arise while planning the deployment of SN: how many sensor nodes have to be deployed to meet certain application purposes and how should the nodes be deployed within the ROI [1].

Optimisation of SN deployment is a process of determining the best possible locations of sensor nodes within the area under study (spatial sampling method). Mamun provided detailed descriptions of existing topologies in wireless sensor networks (WSN), including a comparative discussion of performance of different topologies [2]. As an overview, heuristic-based approaches (mathematical programming) have been extensively utilised to address such NP-hard problem [3]. For instances, evolutionary algorithms ([4], [5]), swarm algorithms [6], linear programming [7], spatial simulated annealing ([8], [9], [10], [11]), and signal processing technique (e.g. wavelet [12]). However, the above-mentioned literature are mainly focusing on the following aspects: coverage of sensing area, network connectivity, and energy consumption. Apart from that, other factors such as cost, spatial analysis and data reconstruction have been described and addressed in ([13], [14]).

In this paper, we present a method to deploy a SN that is able to represent a certain environmental variable of the entire ROI given a certain number of nodes. Section II describes in detail the problem to be addressed in this work, followed by a methodology of the deployment strategy in Section III. Experimental simulation results and discussion is demonstrated in Section IV and conclusions are drawn in Section V.

\section{PROBlem Formulation}

\section{A. Assumption}

The SN design in our work is specifically tailored for weather stations acting as sensor nodes, which are stationary deployed in the ROI. Each node is connected to a telemetry device that enables it to send data to a base station, and is also equipped with a solar panel as an energy source. Therefore, network connectivity and energy consumption are not of concern in this work and are not considered for optimisation.

\section{B. Experimental Dataset}

This study is conducted using the Tasmania's South Esk Hydrological Model, developed by the Commonwealth Scientific and Industrial Research Organisation (CSIRO) [15]. The model covers a set of environmental parameters in the North East of Tasmania $\left(-41.0^{\circ}\right.$ to $-42.0^{\circ}$ latitude and $147.0^{\circ}$ to $148.5^{\circ}$ longitude). The ROI is mapped as a two-dimensional data grid of size of $151 \times 101$ and is in netCDF [16] format. 
A total of one year of averaged daily data are utilised in our experiments. We focused on a number of parameters for different purposes in our study: (a) Elevation data is mainly used for the sensor placements optimisation purpose. Such selection is justified based on the fact that such data can be accessed from a designated source; Tang and collaborators also suggested that high-elevation sample is crucial within the dataset for meteorological studies [17]; (b) Environmental parameters such as temperature, relative humidity, and solar radiation are employed for the evaluation.

\section{Problem Statement}

In this work, the quality of a $\mathrm{SN}$ is measured by the 'degree of representativeness' towards the ROI in which the $\mathrm{SN}$ is deployed. We formulate the representativeness as: how a certain placement of sensor nodes allows an interpolator to best estimate particular environmental variables across the ROI. To achieve a high representativeness of a SN design, we take the following considerations into accounts.

Spatial interpolation is criticised because of the incapability to estimate extreme values [17]. For example, within a mountainous landscape, if we merely deploy the sensors on the lower ground (i.e., foothills in this case), we will not be able to estimate the measurement of interest at the peak of the mountain. Considering such scenario, the focus of our sampling method is to discover the distribution of sensors (encompass those extreme values) to allow best estimation/interpolation of particular environmental phenomena on the later stage. Further, a good interpolator can also be evaluated by the extrapolation capability of distinct methods. Therefore, this issue will also be incorporated in the optimisation criteria.

Towards this end, this paper aims to use EA to optimise the locations of $N$ nodes that minimises the error of the estimated (interpolated and extrapolated) surface under study.

\section{DEPLOYMENT STRATEGY}

The aforementioned problem statement leads us to a multiobjective optimisation problem, where it is typically impossible to have a single solution that satisfies all the objectives. Therefore, the focus is looking for a trade-off amongst the objectives instead of looking for a single solution [18].

\section{A. Evolutionary Algorithm}

We employ an Evolutionary Algorithm (EA) [19] to address the multi-objective optimisation problem in our work. The EA mimics the process of natural selection principles to solve complex searching and optimisation problems. The algorithm starts with a randomly generated population (a set of possible solutions), and it executes the reproduction process in each generation, including, parent selection, crossover, and mutation. In this work, each possible solution (individual) represents a single deployment of $\mathrm{SN}$, which incorporates the position of sensor nodes within the ROI. Elitism is performed at the end of each generation to ensure the best solution seen so far is not lost. Gradually, the most successful individuals evolve to discover the near optimal solutions (Pareto Front) [20].
We utilised EA from a Python library - Distributed Evolutionary Algorithms in Python (DEAP) [21]. Table I presents the parameters that we used in our experiment. These values were chosen from the literature [22]. The process is terminated when the Pareto Front remains the same over the last 50 generation (the stopping criterion).

\begin{tabular}{||rl||}
\hline Parameter & Value \\
\hline Population size & 50 \\
Crossover probability & 0.7 \\
Mutation probability & 0.05 \\
Crossover operation & cxOnePoint \\
Mutation operation & mutUniformInt \\
Selection operation & NSGA2 [23] \\
\hline
\end{tabular}

TABLE I: EA parameters.

\section{B. Fitness Function}

The EA discovers near-optimal solutions according to the so-called fitness functions that define the quality (SN representativeness) of a particular individual (SN design). Let $X=\left\{x_{1}, x_{2}, \ldots, x_{N}\right\}$ be a set of $N$ sensor nodes deployed within the ROI. Based on the objectives of this paper [Section II-C], we formulate the functions as:

1) Fitness Function 1: This function aims to identify the extreme values within the landscape. We leverage the main pitfall of spatial interpolation technique in conjunction with the leave-one-out cross-validation (LOOCV) to assist us in identifying those nodes:

$$
\operatorname{LOOCV}(\hat{f})=\sqrt{\frac{1}{N} \sum_{n=1}^{N}\left(y_{n}-\hat{f}^{(-n)}\left(x_{n}\right)\right)^{2}}
$$

where $\hat{f}$ is a particular interpolation technique [Section III-C]; $y_{n}$ is the observed value at $n^{t h}$ location; and $f^{(-n)}\left(x_{n}\right)$ is the estimated value using $\hat{f}$ with the absent of $n^{t h}$ node (such that $X \backslash\left\{x_{n}\right\}$ ). Then, the fitness function is calculated by maximising such equation, so that an interpolator is able to estimate a good representation of the ROI in the later stage. In other words, this method is trying to find a set of node locations in a way that each node is important and must be deployed within the network. The absence of any node will greatly degrade the representativeness of the area under study.

2) Fitness Function 2: We also want to consider the extrapolation capability of $\hat{f}$ by minimising the root-mean-squarederror (RMSE) of the estimated map's corners:

$$
R M S E(C)=\sqrt{\frac{1}{4} \sum_{n=1}^{4}\left(y_{n}-\hat{f}\left(x_{n}\right)\right)^{2}}
$$

where $C$ is the locations at map's corners that are located at: top-left, top-right, bottom-left and bottom-right of the surface. 


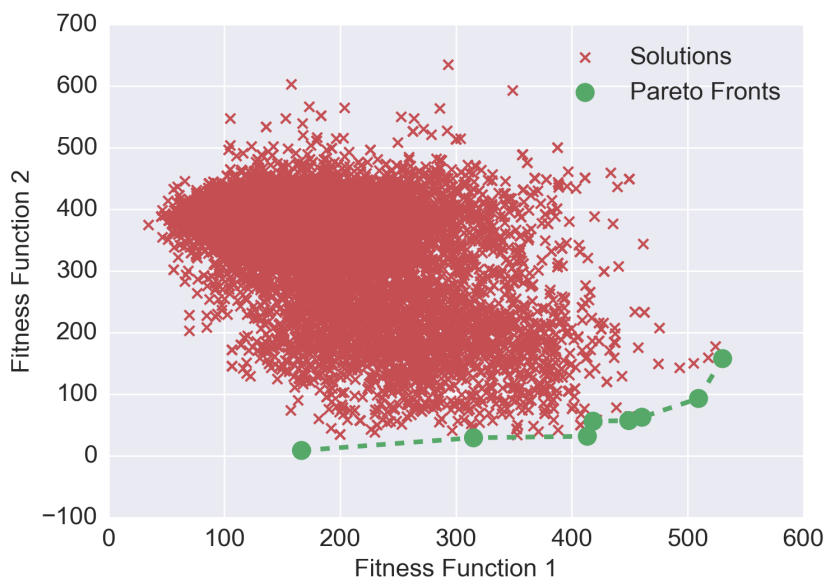

Fig. 1: A typical plotting of multi-objective EA with two objective functions: (a) x-axis, maximisation of Equation 1; and (b) y-axis, minimisation of Equation 2. The red markers are the explored solutions throughout the EA process, and the green dots are the Pareto Front (a list of non-dominated solutions).

\section{Spatial Interpolation Techniques}

This work adopted three of the most frequently used spatial data interpolation techniques [24]: Inverse Distance Weighting (IDW), Ordinary Kriging (OK), and Thin Plate Spline (TPS). We are interested in observing how application of different interpolation techniques on the proposed methodology will affect the performance. Brief description of these methods are:

1) $I D W$ : Utilises the spatial distance between the point to be interpolated and sample points as the main weighting mechanism [25]. This method has been extensively used because it is computationally efficient and produces acceptable results.

2) $O K:$ A geostatistical method that incorporates the local spatial variances of its neighbouring data points within the interpolation process [26]. Kriging-based techniques have been suggested as the optimal method overall from the literature [24], with the downside of being computationally heavy.

3) TPS: A spline-based technique for spatial data interpolation introduced by Duchon in 1976 that passes through each sample point [27]. It is based on the physical analogy involving the bending of a thin metal to create an interpolated surface.

\section{REsults AND Discussions}

In order to evaluate our work, we replicate the simulation using several different runs. While there is no empirical method to determine how many replications are needed, we utilised the method proposed in [28], as follow:

$$
n=\left(\frac{z \times \sigma}{\mu \times a c c}\right)^{2}
$$

where $n$ is the required number of replications, $z$ refers to $z$-score of 1.96 which leads to $95 \%$ of confidence interval; $\mu$ and $\sigma$ are the mean and standard deviation obtained from a preliminary simulations of 10 runs; and the $a c c$ is the percentage of $\mu$ that we want to get as deviation (5\% of accuracy in our case). The following sub-sections are generated using replications based on Table II. We adopt 10 as the minimum number of replications in the case where $n<10$.

\begin{tabular}{cccccccccc}
\hline No. of Nodes & 5 & 10 & 15 & 20 & 25 & 30 & 35 & 40 & 45 \\
\hline OK & 15 & 4 & 6 & 3 & 4 & 1 & 5 & 3 & 3 \\
IDW & 7 & 2 & 2 & 3 & 4 & 2 & 4 & 2 & 1 \\
TPS & 21 & 43 & 27 & 31 & 41 & 56 & 28 & 56 & 67 \\
\hline
\end{tabular}

TABLE II: Number of replications $(n)$ for each respective methods and number of nodes, calculated using Equation 3.

\section{A. The Optimal Interpolator for EA's LOOCV}
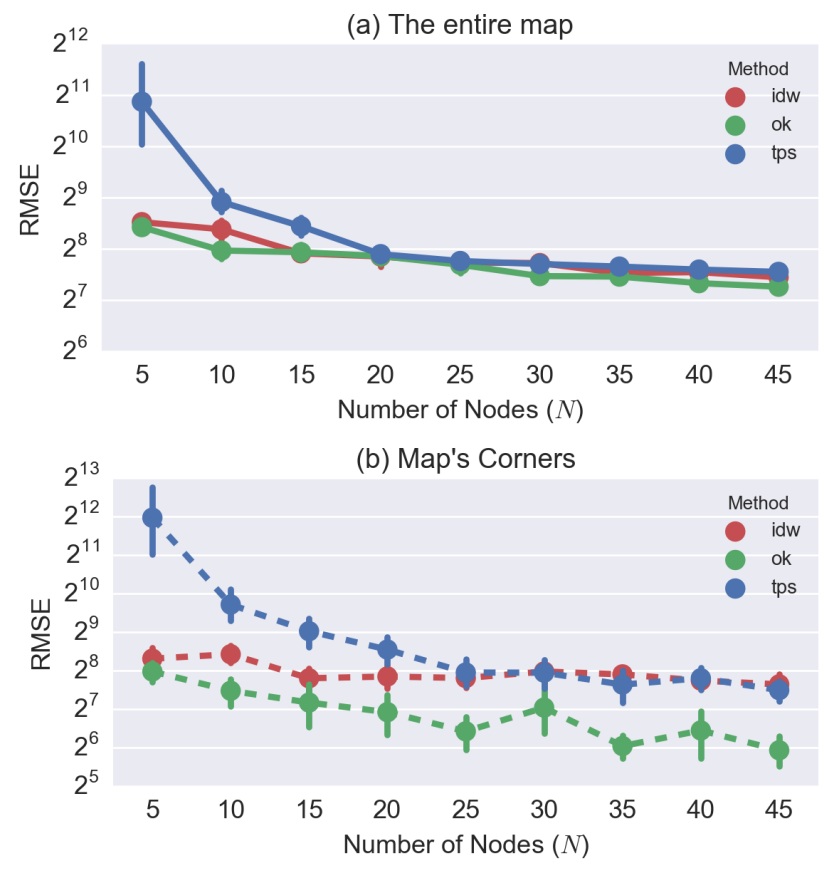

Fig. 2: Interpolation and extrapolation performance of different interpolation techniques. The $\mathrm{x}$-axis represents the number of nodes $(N)$, and y-axis is the RMSE value (the average and $95 \%$ confidence interval). The RMSE is the error calculation between the observed and the estimated surface height data: (a) throughout the landscape; (b) the map's corner.

The first experiment is aimed to determine which interpolation method is best suited for the EA's LOOCV. Since a single run will generate a set of PF that consists of a number of $\mathrm{SN}$ design, we choose the solution that favours the $1^{\text {st }}$ objective function (Eq. 1). In this experiment, we evaluate the techniques based on two criteria:

1 Ability to estimate close-to-reality measurements. RMSE between the observed and the estimated values are calculated throughout the map, and the result is shown in Figure 2a. 
(a) Air temperature

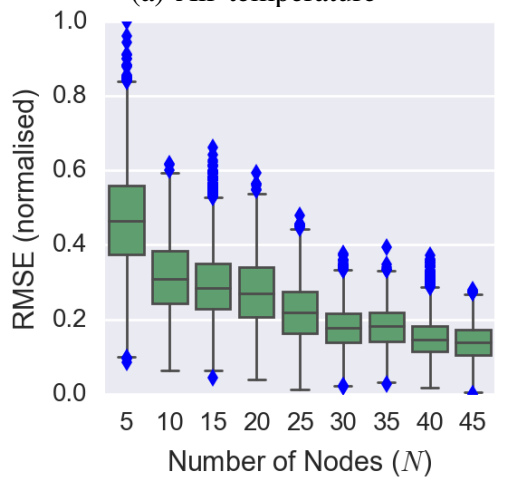

(b) Relative humidity

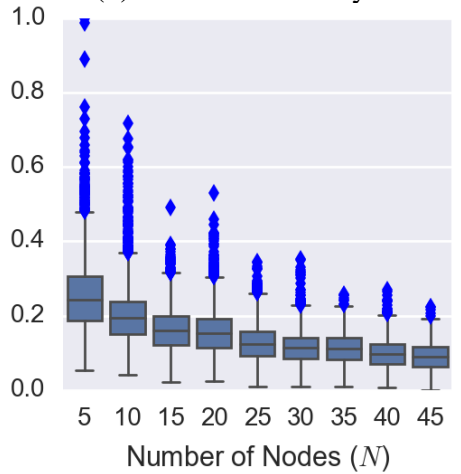

(c) Solar radiation

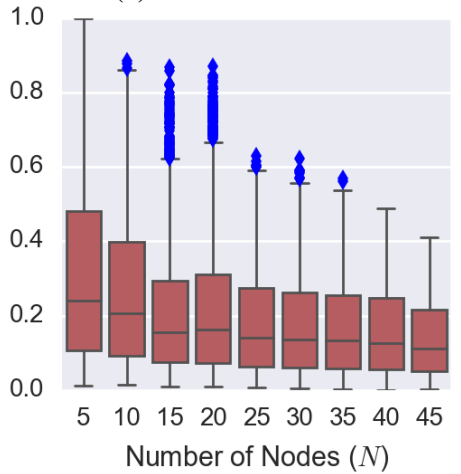

Fig. 3: Performance comparisons among different parameters: (a) air temperature, (b) relative humidity, and (c) solar radiation. The $\mathrm{x}$-axis is the number of nodes, and the $\mathrm{y}$-axis is the normalised RMSE.

2 The 'extrapolation' capability of each method. We calculated the RMSE at the map's corner. The result is shown in Figure $2 b$.

Figure 2a presents the performance amongst three compared spatial interpolation techniques: OK, IDW, and TPS. The result shows that OK performs the best (lowest RMSE), and it is followed by IDW and TPS. The confidence interval (CI) of OK and IDW are relatively small that are barely noticeable, which indicates that both methods produce relatively stable results. TPS on the other hand, has a very large CI with number of nodes $(N)$ being 5 , which becomes less significant as $N$ increases. The performance among these methods progress to converge as $N=20$; further increase in $N$ does not substantially reduce the RMSE.

According to Figure 2b, the extrapolating capabilities of the compared techniques has the similar performance behaviour as in Figure 2a (OK, followed by IDW and TPS). Interestingly, the extrapolating performance between IDW and TPS becomes comparable starting from $N=25$, where IDW performs relatively stable regardless of $N$. The fact that OK outperforms other techniques is not surprising, considering OK incorporates geostatistical analysis within its calculation.

These results have shown that OK suits the best for our proposed method. Thus, the subsequent simulation will utilise $\mathrm{OK}$ as the interpolation technique within EA's LOOCV.

\section{B. Performance Assessment}

This simulation is used to validate the proposed method by comparing the interpolation RMSE generated from the sampling design of different environmental parameters: temperature, relative humidity, and solar radiation. The main objective of this experiment is to determine whether using surface height data within the optimisation (spatial sampling design) is able to generate a close-to-reality measurement of other parameters within the ROI.

Figure 3 demonstrates the simulation results. In general, it shows that the RMSE decreases for each parameter as the number of nodes $N$ increases. However, it is noticeable that air temperature has the most significant quality improvement as $N$ increases [Figure 3a], as reflected in median, interquartile range (IQR), whiskers, and outliers. It is then followed by relative humidity [Figure 3b] that behaves similarly with temperature (median), while the only discrepancy is that less significant improvement in terms of whiskers and outliers. Finally, for the case of solar radiation [Figure 3c], a slight improvement can be observed for the median, but not the variability, especially whiskers and outliers (e.g., $N=20$ ).

As a result, under current empirical study, we suggest that using elevation in sampling design suffices to obtain confident temperature and relative humidity data. In a less restricted budget situation, best representativeness of all the variables could be achieved by employing 35 nodes (e.g., Figure 4).

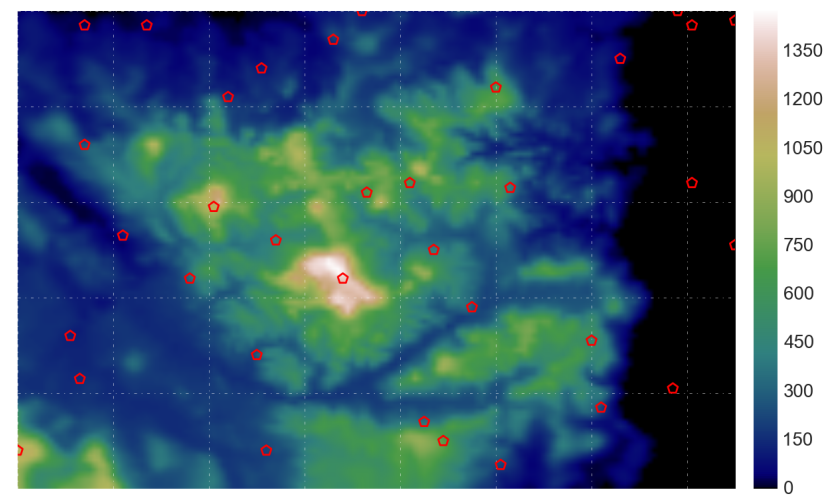

Fig. 4: An example of a sensor network deployment (35 sensor nodes) within the ROI, generated by the proposed methodology. This figure demonstrates the elevation map from the SouthEsk model, and the red dots are the node locations to be deployed within the landscape. 


\section{CONCLUSIONS}

The main objective of this paper is to obtain near optimal sensor node placements that allow any interpolator to best estimate a particular environmental phenomena at interest. A novel spatial sampling design approach is proposed by using multi-objective Evolutionary Algorithm (EA) to minimise the prediction error [Section III-B].

In order to define which interpolation technique is suitable for our work, three different spatial interpolation techniques are compared. Our results indicate that using Ordinary Kriging produces the best results, which yields the lowest interpolation error and best extrapolating capability [Section IV-A].

This work provides a simulation which would help decision makers while designing a SN that could deliver fit-for-purpose data without introducing undesirable costs resulting from excessive placement of nodes. Based on the outputs (Pareto optimality solutions) generated by the proposed method [Figure 1], the decision makers are required to select single SN design that incorporates additional consideration of their domain knowledge (i.e., sparsity and the feasibility of the deployment) that is suitable for their purpose.

\section{ACKNOWLEDGEMENT}

Ferry Susanto would like to thank Victoria University and Vale Institute of Technology for the the $\mathrm{PhD}$ scholarship, and CSIRO for the "Office of the Chief Executive" top-up scholarship. Setia Budi would like to thank Sense-T for the $\mathrm{PhD}$ scholarship and CSIRO for the top-up scholarship.

We also want to express our gratitude to the anonymous reviewers who have given us excellent comments that greatly improved the contents of this work.

\section{REFERENCES}

[1] E. Onur, C. Ersoy, and H. Deliç, "How many sensors for an acceptable breach detection probability?" Computer Communications, vol. 29, no. 2, pp. 173-182, Jan. 2006.

[2] Q. Mamun, "A Qualitative Comparison of Different Logical Topologies for Wireless Sensor Networks," Sensors, vol. 12, no. 11, pp. 14887 14913, Nov. 2012.

[3] M. Younis and K. Akkaya, "Strategies and techniques for node placement in wireless sensor networks: A survey," Ad Hoc Networks, vol. 6, no. 4, pp. 621-655, Jun. 2008

[4] M. Mansouri, H. Nounou, and M. Nounou, "Genetic Algorithm-based Adaptive Optimization for Target Tracking in Wireless Sensor Networks,' Journal of Signal Processing Systems, vol. 74, no. 2, pp. 189 202, Jun. 2013

[5] S. Budi, P. de Souza, G. Timms, V. Malhotra, and P. Turner, "Optimisation in the design of environmental sensor networks with robustness consideration," Sensors, vol. 15, no. 12, p. 29765, 2015 [Online]. Available: http://www.mdpi.com/1424-8220/15/12/29765

[6] R. Kulkarni and G. Venayagamoorthy, "Particle Swarm Optimization in Wireless-Sensor Networks: A Brief Survey," IEEE Transactions on Systems, Man, and Cybernetics, Part C: Applications and Reviews, vol. 41, no. 2, pp. 262-267, Mar. 2011.

[7] M. Rebai, M. Le berre, H. Snoussi, F. Hnaien, and L. Khoukhi, "Sensor deployment optimization methods to achieve both coverage and connectivity in wireless sensor networks," Computers \& Operations Research, vol. 59, pp. 11-21, Jul. 2015.
[8] W. Jianghao, G. Yong, G. B. M. Heuvelink, and Z. Chenghu, "Spatial sampling design for estimating regional gpp with spatial heterogeneities," Geoscience and Remote Sensing Letters, IEEE, vol. 11, no. 2 , pp. 539-543, 2014.

[9] G. B. M. Heuvelink, Z. Jiang, S. De Bruin, and C. J. W. Twenhöfel, "Optimization of mobile radioactivity monitoring networks," International Journal of Geographical Information Science, vol. 24, no. 3, pp. 365-382, 2010.

[10] Y. Ge, J. H. Wang, G. B. M. Heuvelink, R. Jin, X. Li, and J. F. Wang, "Sampling design optimization of a wireless sensor network for monitoring ecohydrological processes in the babao river basin, china," International Journal of Geographical Information Science, vol. 29, no. 1 , pp. 92-110, 2015.

[11] J. Kang, X. Li, R. Jin, Y. Ge, J. Wang, and J. Wang, "Hybrid optimal design of the eco-hydrological wireless sensor network in the middle reach of the heihe river basin, china," Sensors (Basel), vol. 14, no. 10, pp. 19095-114, 2014.

[12] A. L. L. Aquino, R. A. R. Oliveira, and E. F. Wanner, "A waveletbased sampling algorithm for wireless sensor networks applications," pp. 1604-1608, 2010.

[13] A. Frery, H. S. Ramos, J. Alencar-Neto, E. Nakamura, and A. A. F. Loureiro, "Data driven performance evaluation of wireless sensor networks," Sensors, vol. 10, no. 3, p. 2150, 2010.

[14] C. D’Este, P. d. Souza, C. Sharman, and S. Allen, "Relocatable, Automated Cost-Benefit Analysis for Marine Sensor Network Design," Sensors, vol. 12, no. 3, pp. 2874-2898, Mar. 2012.

[15] J. Katzfey and M. Thatcher, "Ensemble one-kilometre forecasts for the South Esk Hydrological Sensor Web," 19th International Congress on Modelling and Simulation (modsim2011), pp. 3511-3517, 2011, wOS:000314989303069.

[16] R. Rew and G. Davis, "NetCDF: an interface for scientific data access," IEEE Computer Graphics and Applications, vol. 10, no. 4, pp. 76-82, Jul. 1990.

[17] L. Tang, X. Su, G. Shao, H. Zhang, and J. Zhao, "A clustering-assisted regression (car) approach for developing spatial climate data sets in china," Environmental Modelling and Software, vol. 38, no. 0, pp. 122$128,2012$.

[18] K. Deb, Multi-Objective Optimization Using Evolutionary Algorithms, 1st ed. Chichester; New York: Wiley, Mar. 2009.

[19] C. C. Coello, G. B. Lamont, and D. A. v. Veldhuizen, Evolutionary Algorithms for Solving Multi-Objective Problems, 2nd ed. New York: Springer, Sep. 2007

[20] Y. Censor, "Pareto optimality in multiobjective problems," Applied Mathematics and Optimization, vol. 4, no. 1, pp. 41-59, Mar. 1977.

[21] F.-A. Fortin, F.-M. De Rainville, M.-A. G. Gardner, M. Parizeau, and C. Gagné, "DEAP: Evolutionary Algorithms Made Easy," J. Mach. Learn. Res., vol. 13, no. 1, pp. 2171-2175, Jul. 2012.

[22] M. Srinivas and L. Patnaik, "Genetic algorithms: a survey," Computer, vol. 27, no. 6, pp. 17-26, June 1994.

[23] K. Deb, A. Pratap, S. Agarwal, and T. Meyarivan, "A fast and elitist multiobjective genetic algorithm: NSGA-II," IEEE Transactions on Evolutionary Computation, vol. 6, no. 2, pp. 182-197, Apr. 2002.

[24] J. Li and A. D. Heap, "A review of comparative studies of spatial interpolation methods in environmental sciences: Performance and impact factors," Ecological Informatics, vol. 6, no. 3-4, pp. 228-241, 2011.

[25] D. Shepard, "A two-dimensional interpolation function for irregularlyspaced data," pp. 517-524, 1968.

[26] P. A. Burrough and R. A. McDonnell, "Principles of geographical information systems," 1998.

[27] J. Duchon, Splines minimizing rotation-invariant semi-norms in Sobolev spaces, ser. Lecture Notes in Mathematics. Springer Berlin Heidelberg, 1977, vol. 571, book section 7, pp. 85-100.

[28] R. Jain, The Art of Computer Systems Performance Analysis: Techniques 
for Experimental Design, Measurement, Simulation, and Modeling.

Wiley, 1991. 